[0212-7199 (2007) 24: 10; pp 497-499] ANALES DE MEDICINA INTERNA Copyright (C) 2007 ARAN EDICIONES, S.L. AN. MED INTERNA (Madrid) Vol. 24, N. ${ }^{\circ} 10$, pp. 497-499, 2007

\section{Histiocitosis de células de Langerhans con afectación tímica como manifestación única de la enfermedad}

\author{
J. M. HERNÁNDEZ PÉREZ, T. FRANQUET CASAS ${ }^{1}$, S. RODRÍGUEZ ${ }^{1}$, A. GIMÉNEZ'

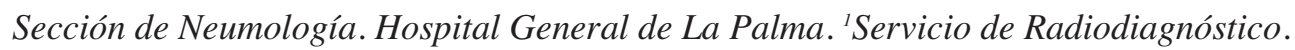 \\ Hospital de la Santa Creu i Sant Pau. Universidad Autónoma de Barcelona
}

THE LANGERHANS' CELL HISTIOCYTOSIS WITH THYMIC LOCALIZATION AS INITIAL AND EXCLUSIVE PLACE

\begin{abstract}
RESUMEN
La histiocitosis de células de Langerhans (HCL), conocida también como Histiocitosis $\mathrm{X}$ es una enfermedad granulomatosa poco frecuente de etiología no aclarada que puede tener manifestaciones y localizaciones diversas, sin embargo la localización tímica como lugar inicial y exclusivo de presentación de HCL es bastante inusual. Se presenta el caso de un paciente que debutó con una clínica inespecífica, donde las pruebas de imagen pusieron de manifiesto una masa en mediastino anterior y que tras los análisis anatomopatológicos e inmunohistoquímicos evidenciaron una proliferación de células de Langerhans y eosinófilos siendo positiva para CD1a y S-100 confirmando el diagnóstico de HCL.
\end{abstract}

PALABRAS CLAVE: Histiocitosis X. Histiocitosis de células de Langerhans. Timo.

\begin{abstract}
The Langerhans'cell histiocytosis ( $\mathrm{LCH})$, also known as Histiocitosis $X$ it is an illness not very frequent granulomatosus etiology not clarified yet, that it can have different manifestations and localizations, however the thymic localization as initial and exclusive place gives presentation $H C L$ it is quite unusual. The present case is presented a patient that debuted with a clinical unspecific, where the tests give image they put she gives apparent a mass in previous mediastinum and that after the pathologic and immunohistochemical analysis they evidenced a proliferation Langerhans's cells and eosinophils it being positive for CDIa and S-100 confirming the diagnosis of the $\mathrm{LCH}$.
\end{abstract}

KEY WORDS: Histiocytosis X. Langerhans'cell histiocytosis. Thymus.

Hernández Pérez JM, Franquet Casa T, Rodríguez S, Giménez. A. Histocitosis de células de Langerhans con afectación tímica como manifestación única de la enfermedad. An Med Interna (Madrid) 2007; 24: 497-499.

\section{INTRODUCCIÓN}

La HCL comprende un espectro de diferentes enfermedades donde se encuentran el granuloma eosinófilo, la enfermedad de Letterer-Siwe y la enfermedad de Hand-SchullerChristian (1). En la mayoría de ocasiones suele debutar en niños y gente joven siendo la localización más frecuente de afectación ósea y, con menor frecuencia, piel, pulmón y multiorgánica. Su etiología no está clara aunque se ha visto en relación con neoplasias de origen hematológicas (2) (tipo linfoma no Hodgkin y enfermedad de Hodgkin), adenocarcinomas (pulmón y mama) y excepcionalmente con sarcomas (3), aunque también se describen asociaciones con enfermedades autoinmunes como la miastenia gravis $(2,4)$.

En localizaciones como la piel, las manifestaciones clínicas son más llamativas y el diagnóstico de sospecha sobreviene de manera más rápido, sin embargo en otras como pulmón, debemos apoyarnos en pruebas de imagen (tomografía computerizada (TC) - tomografía de alta resolución) que al encon- trarnos con la presencia de quistes redondos, bilobulados o ramificados localizados en zonas medias y superiores, junto la existencia de nódulos de pequeño tamaño el diagnóstico de HCL se hace más que probable, aún así la confirmación sólo se puede obtener por métodos anatomopatológicos e inmunohistoquímicos (5). Su tratamiento no está estandarizado, y puede ir desde la mera expectación (remiten espontáneamente en un gran número de casos), hasta llegar a utilizar agentes quimioterápicos e incluso trasplantes de médula ósea. La supervivencia de esta enfermedad se sitúa en torno al $90 \%$, pero a pesar de ello se precisa de un seguimiento estricto de los pacientes debido a su potencial de diseminación o recidiva.

\section{CASO APORTADO}

Se trata de un varón de 19 años de edad que ingresa refiriendo clínica consistente en dolor intermitente localizado a nivel del hemotórax izquierdo acompañado de tos y febrícula de un mes de evolu-

Trabajo aceptado: 31 de mayo de 2007

Correspondencia: José María Hernández Pérez. Sección de Neumología. Hospital General de La Palma. C/ Buenavista de Arriba, s/n. 38710 Breña Alta. Isla de La Palma.e-mail: jmherper@terra.es 
ción. En la analítica general practicada solo llamó la atención la existencia de una leucocitosis de $16.500 \times 10^{9} / 1$ y una VSG de $24 \mathrm{~mm}$ en $1 \mathrm{~h}$, el resto de pruebas complementarias incluyendo microbiológicas fueron negativas. En la radiografía de tórax practicada se objetivaba una masa de bordes irregulares que borraba el contorno del hilio pulmonar izquierdo (Fig. 1), tras realizarse la TC de tórax se confirmó la existencia de una masa de bordes más nítidos con áreas hipodensas en su interior localizada en mediastino anterior y en contacto íntimo con estructuras cardíacas de 10 × $6 \mathrm{~cm}$ de diámetro (Fig. 2). La biopsia obtenida por videotoracoscopia de la masa manifestó que estaba constituida por una proliferación de células de Langerhans con abundantes eosinófilos, las tinciones histoquímicas realizadas mostraron positividad para S-100 y CD1a, siendo negativas para marcadores linfoides, todos estos hallazgos fueron compatibles con el diagnóstico de HCL. Tras recibir 2 líneas de tratamiento quimioterápico y sin obtener respuesta evidente, fue necesario iniciar terapia radioterápica, encontrándose durante la misma la aparición de una adenopatía en localización supraclavicular, que tras ser biopsiada se evidenció enfermedad de Hodgkin tipo esclerosis nodular asociada a HCL; tras no observarse respuesta al tratamiento convencional, se llevó a cabo autotrasplante de precursores hematopoyéticos de sangre periférica, consiguiendo remisión completa de ambas enfermedades hasta la fecha.

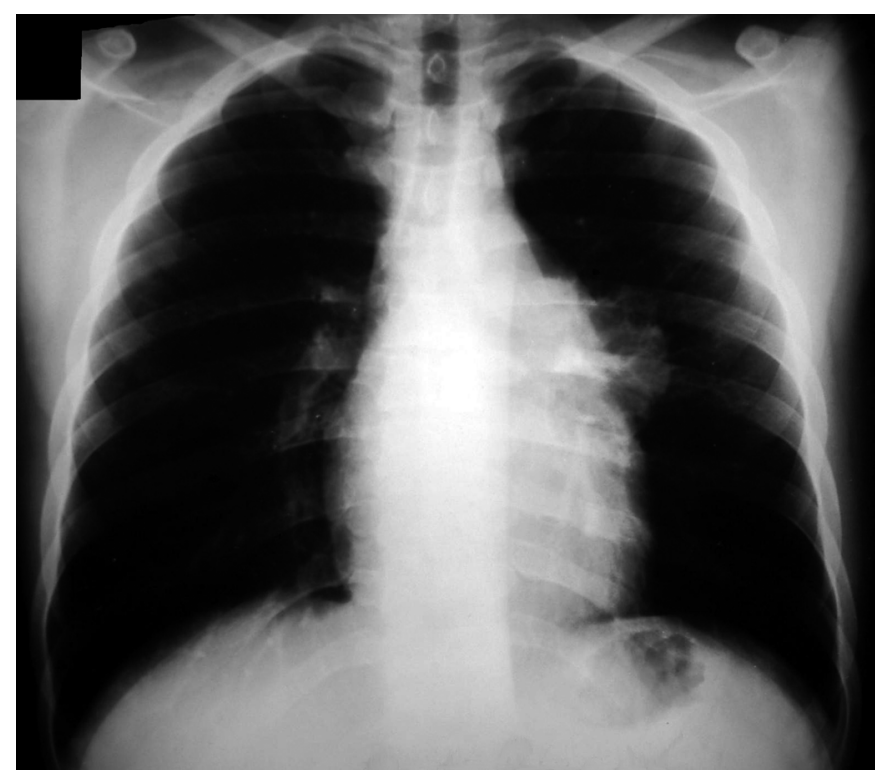

Fig. 1. Lesión de contornos irregulares que hace signo de la silueta con el hilio izquierdo alterando su disposición normal.

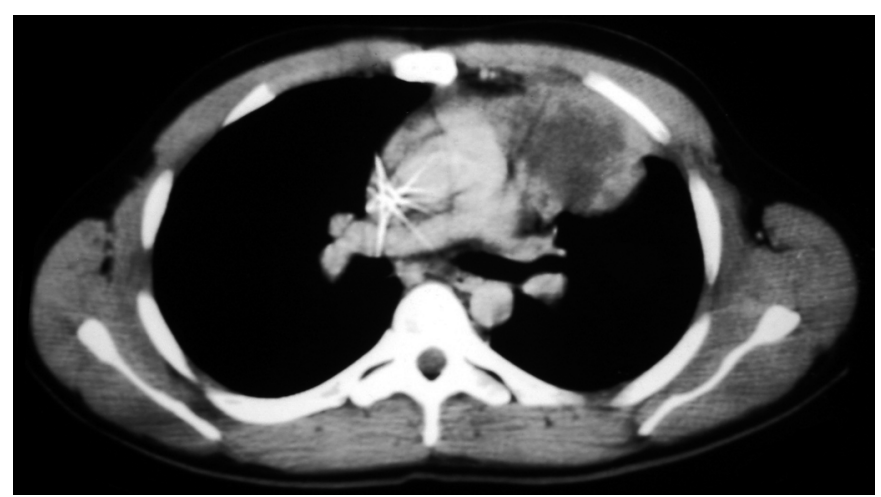

Fig. 2. Masa de bordes nítidos con áreas hipodensas en su interior, localizada en mediastino anterior que contacta con estructuras cardíacas.

\section{DISCUSIÓN}

La HCL fue descrita por primera vez en 1939 por Andrews y a pesar de haber pasado más de 60 años desde su primera descripción, su etiología aún no ha sido desvelada (6). Debido a que se ha visto en relación con neoplasias hematológicas como presenta nuestro caso, es fácil pensar de que ambas enfermedades deben tener un origen común a nivel de producción anómala de clones de células y por ello considerar a la HCL hasta cierto punto, como una enfermedad neoplásica; sin embargo la resolución en ocasiones de la enfermedad sin tratamiento y la respuesta favorable a tratamiento con corticoides en otras, va en contra de este sentido.

A pesar de que la localización del timo, en la enfermedad HCL multiorgánica es frecuente (7), el timo como manifestación inicial y única de la HCL es bastante inusual, así lo atestiguan solo 11 descripciones anteriores en la literatura (Tabla I), donde encontramos algunas similitudes, como la asociación con la miastenia gravis y neoplasias diversas, así como la aparición en la TC de tórax la presencia de múltiples calcificaciones intratímicas dispersas pretratamiento (8-10;) aunque en el caso expuesto no se aprecien dichos hallazgos en la TC, quizás sea debido a esto al rápido crecimiento de la lesión y a la prontitud de las manifestaciones clínicas, no dando tiempo tal vez, a la formación de dichas calcificaciones y por tanto a su observación en las pruebas de imagen.

\section{TABLA}

REVISIÓN DE AUTORES Y CASOS DE AFECTACIÓN TÍMICA POR HCL EN LA LITERATURA

\begin{tabular}{|c|c|c|c|c|c|}
\hline Autor & $\begin{array}{l}N^{0} \text { de } \\
\text { casos }\end{array}$ & $\begin{array}{c}\text { Enfermedad } \\
\text { asociada }\end{array}$ & Calcificaciones & s Tratamiento & Evolución \\
\hline Beatty13 & 1 & No & 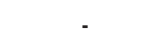 & Cirugía + RT & Favorable \\
\hline \multirow[t]{2}{*}{ Siegal et al14 } & 2 & No & - & Cirugía + RT & Favorable \\
\hline & & No & & Cirugía + QT & Favorable \\
\hline Pescarmona et al11 & 1 & Miastemia C. & No & Cirugía & Favorable \\
\hline Bramwell and Burns2 & 1 & Miastemia C. & No & Ciruǵa + Esteroides & Favorable \\
\hline Cilcrease4 & 1 & Miastemia C. & 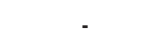 & Cirugía & Favorable \\
\hline Wakely et al5 & 1 & No & $\mathrm{Si}$ & Ninguno & Favorable \\
\hline Elliot et al6 & 1 & No & $\mathrm{Si}$ & Ninguno & Favorable \\
\hline Novak et al12 & 1 & T. de Fallot & - & Ninguno & Favorable \\
\hline Lee et al3 & 1 & Sarcoma & No & Cirugía & Favorable \\
\hline Presente caso & 1 & Enf. hodgkin & No & QT+RT+TAPSP & Favorable \\
\hline
\end{tabular}

RT: radioterapia; QT: quimioterapia; TAPSP: trasplante autólogo de precursores de sangre periférica, - No se realizó TC.

Debido a la gran diversidad de expresión clínica de esta enfermedad y a la impredecible evolución de ésta, es difícil la elección terapeútica, sin embargo en algunos casos la opción está muy clara, como puede ser el caso de la afectación multiorgánica, donde la quimioterapia parece ser el tratamiento de elección; pero en aquellas localizaciones únicas como es el caso expuesto, no queda claro que actitud llevar a acabo, así queda reflejado en al literatura donde hay casos tratados exclusivamente con cirugía $(3,11)$, otros con resolución espontánea y otros como el presente caso donde fue necesario llegar al trasplante de médula ósea, si bien cabe destacar que al estar asociado a una enfermedad de Hodgkin el tratamiento debía ser más intensivo. 
El hecho de que en ocasiones esta localización haya sido un hallazgo meramente casual (12), nos da que pensar que muchas veces haya podido pasar desapercibido y por tanto su diagnóstico haya quedado subestimado, de ahí que solo con el caso expuesto nos encontremos con tan pocas referencias dentro de la literatura; por tales motivos, nos hace pensar que a priori el hallazgo de una masa mediastínica anterior en un paciente joven, hace que el diagnóstico diferencial se base sobre todo en timomas, linfomas, teratomas y patología tiroidea, pero que junto al hallazgo de calcificaciones en la TC y más aún la existencia de miastenia gravis o neoplasia asociada, debe llevarnos a incluir, a pesar de su infrecuencia, la HCL como posibilidad diagnóstica, siendo necesaria de la confirmación anatomopatológica e inmunohistoquímica posterior.

\section{Bibliografía}

1. Martínez J, Pardo N, Torrent M, Moliner E, Anquela L, Cubells J. Histiocitosis de células de Langerhans: diferentes manifestaciones de una misma base histopatogénica. An Esp Pediatr 2002; 57: 484-7.

2. Bramwell NH, Burns BF. Histyocitosis $X$ of the thymus in association with myasthenia gravis. Am J Clin Pathol 1986; 86: 223-7.

3. Lee BH, George S, Kutok JL. Langerhans cell histiocytosis involving the thymus. A case report and review of the literature. Arch Pathol Lab Med 2003; 127: 294-7.

4. Gilcrease MZ, Rajan B, Ostrowski ML, Ramzy L, Schwartz MR thymic Langerhans'cell histiocytosis and its relationship with myasthenia gravis. Immunohistochemical, ultrastructural, and cytometric studies. Arch Pathol Lab Med 199; 121: 134-8.

5. Wakely P Jr, Suster S. Langerhans'cell histiocytosis of the thymus associated with multilocular thymic cyst. Hum Pathol 2000; 31: 1532-5.

6. Elliott M, Kokai GK, Abemethy LJ, Pizer BL. Spontaneous resolution of isolated thymic Langerhans cell histyocytosis. Med Pediatr Oncol 12002; 38: 274-6.

7. Newton WA Jr, Hamoudi AB, Shannon BT. Role of the thymus in histi- ocytosis X. Hematol Oncol Clin North Am 1987; 1: 63-7.

8. Junewick JJ, Fitzgerald NE. The thymus in Langerhans'cell histiocytosis. Pediatr Radial 1999; 29: 904-7.

9. Heller GD, Haller JO, Berdon WE, Sane S, Kleinman PK. Punctate thymic calcification in infants with untreated Langerhans'cell histiocytosis: Report of four new cases. Pediatr Radial 1999; 29: 813-5.

10. Sumner TE, Auringer ST, Prestan AA. Thymic calcifications in histiocytosis X. Pediatr Radial 1993; 23: 204-5.

11. Pescarmona E, Redina EA, Ricci C, Baroni CD. Histyocitosis X and lymphoid follicular hyperplasia of the thymus in myasthenia gravis. Histopathol 1989; 14: 465-70.

12. Novak L, Castro CY, Listinky CM. Multiple Langerhans'cell nodules in an incidental thymectomy. Arch Pathol Lab Med 2003;127: 218-20.

13. Beatty EC Jr. Eosinophilic granuloma of parotid gland and thymus. AJDC 1963; 105: 119-22.

14. Siegal GP, Dehner LP, Rosai J. Histiocytosis X (Langerhans'cell granulomatosis) of the thymus. A clinicopathologic study of four childhood cases. Am J Surg Pathol 1985; 9: 117-24. 